\title{
Producción española en biblioteconomía y documentación con visibilidad internacional a través del Web of science (1995-2004)
}

\author{
Por Evaristo Jiménez Contreras, Emilio Delgado López-Cózar y Rafael Ruiz Pérez
}

\begin{abstract}
Jiménez Contreras, Evaristo; Delgado López-Cózar, Emilio; Ruiz Pérez, Rafael. «Producción española en biblioteconomía y documentación con visibilidad internacional a través del Web of science (1995-2004)». En: El profesional de la información, 2006, septiembre-octubre, v. 15, n. 5, pp. 373-383.
\end{abstract}

\section{EL USO GENERALIZADO}

DEL WOS (Web of science) -producto sintetizador de las ya clásicas bases de datos Science citation index, Social sciences citation index y Arts \& humanities citation index- para evaluar el rendimiento investigador de los científicos españoles ha conducido en los últimos años a la proliferación de estudios bibliométricos cuyo propósito más o menos explícitamente declarado es fijar escalafones de productividad, visibilidad y repercusión a partir del recuento de publicaciones y citas recibidas por científicos e instituciones.

La reciente publicación en esta revista de varios artículos breves a cargo del grupo Scimago (2005, 2006) obedece en última instancia a este fenómeno, más si cabe teniendo en cuenta que no ha más de tres años se publicaba en $B i D$ un trabajo (Jiménez, 2002) con el mismo objetivo, similar factura y casi idéntica cobertura (1992-2001 frente a 1995-2004). Estos dos trabajos son un buen botón de muestra de una circunstancia que pende siempre sobre los estudios bibliométricos y que puede ensombrecer sus virtudes y dañar la credibilidad en los mismos. A saber: planteándose igual propósito los resultados obtenidos, sin embargo, difieren, y no de forma cosmética sino sustantiva. $Y$ es que nunca debemos olvidar algo que es obvio: los resultados de un estudio bibliométrico son directamente proporcionales a la fuente de datos empleada y, especialmente, a la estrategia de búsqueda y recopilación de datos aplicada.
Pretende esta contribución reexaminar la metodología sobre la que ésos se asientan valorando las limitaciones de las recuperaciones basadas sólo en categorías ISI, comparar los resultados con los obtenidos a partir de búsquedas temáticas y con los de las afiliaciones de los autores $\mathrm{y}$, por último, revisar igualmente los datos de la producción e impacto de los autores más citados para refinar sus resultados en ambos ámbitos, igualmente mediante estrategias de búsqueda tradicionales, no automáticas, que, como se demuestra, incrementan notablemente la recuperación.

\section{Una cosa es la categoría ISLS y otra los artículos}

Según reza en el título del estudio de Scimago, y se declara repetidamente a lo largo del texto, se pretende identificar la producción española con visibilidad internacional en ByD (biblioteconomía y documentación), entendiendo por tal aquella que figura en la base de datos WOS del ISI (Institute for Scientific Information), hoy Thomson Scientific. La realidad es que el estudio se limita estrictamente a identificar la producción publicada por científicos españoles en las 54 revistas indizadas (hoy día 60 y a lo largo de estos últimos años unas 100) en la categoría ISLS (information science and library science) del WOS o la aparecida en aquellos artículos que, publicados en revistas alojadas en otras categorías temáticas del WOS, tengan a las revistas de ISLS como las más citadas en sus referencias bibliográficas.
Pues bien, este planteamiento se edifica sobre una premisa que es conceptualmente discutible. A saber: sólo se considera que un trabajo es de ByD si se ha publicado en [o ha citado mayoritariamente a] las revistas que figuran en una de las categorías temáticas en las que se ha organizado la literatura científica cubierta por una determinada base de datos. Es decir, es la clasificación en categorías temáticas realizada por los responsables del WOS la que determina el tema de los artículos y no al revés, como sería lógico. Evidentemente, la naturaleza y el contenido de un trabajo no dependen del medio en que éste se publique, aunque en un principio contenido y medio debieran, o pudieran, guardar relación, sino de su propia sustancia temática. En definitiva, un trabajo es de ByD porque trata un tema que le es propio a esta disciplina y no porque se publique en tal o cual revista.

\section{«Un trabajo es de ByD porque trata un tema que le es propio a esta disciplina y no porque se publique en tal o cual revista»}

No debemos olvidar que ni las publicaciones, y menos aún las categorías temáticas, pueden considerarse compartimentos estancos equivalentes a las secciones en que se estructuran unos grandes almacenes. Es evidente que las categorías se componen de revistas que son bastante heterogéneas entre sí; y 


\begin{tabular}{|c|c|c|c|c|c|c|}
\hline \multirow{2}{*}{$\begin{array}{l}\text { Número de } \\
\text { categorías } \\
\text { temáticas } \\
\text { asignadas a } \\
\text { las revistas }\end{array}$} & \multicolumn{2}{|c|}{$\begin{array}{c}\text { WOS-2004 } \\
\text { Information science } \\
\text { and library science }\end{array}$} & \multicolumn{2}{|c|}{$\begin{array}{l}\text { WOS-2004 } \\
\text { Psychology }\end{array}$} & \multicolumn{2}{|c|}{ JCR-Science 2002} \\
\hline & No revistas & $\%$ & No revistas & $\%$ & No revistas & $\%$ \\
\hline 1 & 30 & 55,6 & 326 & 74,3 & 3.332 & 56,7 \\
\hline 2 & 17 & 31,5 & 84 & 19,1 & 1.814 & 30,9 \\
\hline 3 & 6 & 11,1 & 24 & 5,5 & 601 & 10,2 \\
\hline 4 & 1 & 1,8 & 5 & 1,1 & 120 & 2,0 \\
\hline 5 & - & - & - & - & 8 & 0,1 \\
\hline 6 & - & - & - & \begin{tabular}{|l}
- \\
\end{tabular} & 1 & 0,0 \\
\hline Total & 54 & 100,0 & 439 & 100,0 & 5.876 & 100,0 \\
\hline
\end{tabular}

Tabla 1. Distribución del número de categorías temáticas asignadas a las revistas indizadas en diversos campos del WOS

que éstas, incluso en sí mismas, constituyen auténticos conglomerados formados por la adición de artículos singulares que pueden tratar de múltiples temáticas. Buena prueba de que su contenido no es monolítico es lo habitual que resulta en el WOS la conocida práctica de clasificación simultánea de una revista en varias categorías. Los datos al respecto son concluyentes: el $44,4 \%$ de los títulos de publicaciones indizadas bajo la ISLS (tabla 1) figuran en dos o más categorías temáticas del WOS, porcentaje ligeramente superior al existente en todo el campo científico-tecnológico (Science citation index) y muy por encima del $26 \%$ que presenta, por ejemplo, la psicología, una disciplina también del ámbito de las ciencias sociales que es donde se ubica la ByD.

Centrando el análisis en ISLS, que es lo que aquí nos interesa, basta leer la definición que ofrece el
WOS de la misma para que los especialistas en ByD nos demos cuenta de lo laxa y poco precisa que resulta: "information science \& library science covers resources on a wide variety of topics, including bibliographic studies, cataloguing, categorization, database construction and maintenance, electronic libraries, information ethics, information processing and management, interlending, preservation, scientometrics, serials librarianship, and special libraries".

http://www.isinet.com/journals/sco pe/scope_scie.html

Puede resultar anecdótico pero sólo en dos categorías del Social sciences citation index (ISLS y Social issues) los responsables del WOS se curan en salud declarando que la categoría cubre recursos "on a wide variety of topics". Por otra parte, podemos apreciar cuáles son las categorías temáticas más empleadas, además de la propia ISLS, pa-

\begin{tabular}{|l|c|}
\hline $\begin{array}{l}\text { Otras categorías no ISLS asignadas a } \\
\text { revistas ISLS }\end{array}$ & N. \\
\hline Computer science, information systems & 16 \\
\hline Computer science, interdisciplinary applications & 3 \\
\hline Management & 3 \\
\hline Communication & 2 \\
\hline Geography & 1 \\
\hline Humanities, multidisciplinary & 1 \\
\hline Law & 1 \\
\hline Medical informatics & 1 \\
\hline Multidisciplinary sciences & 1 \\
\hline Social sciences, interdisciplinary & 1 \\
\hline Telecommunications & 1 \\
\hline
\end{tabular}

Tabla 2. Diferentes categorías del WOS en las que se encuentran indizadas las revistas de information science and library science ra clasificar las revistas de ISLS (tabla 2). Su lectura nos dará una idea las áreas consideradas por esta base de datos como más próximas a la nuestra.

Además, conviene resaltar que el hecho de incluir una revista en una categoría temática no la convierte automáticamente en un medio de comunicación exclusivo de la comunidad científica y profesional de dicho campo. Casi un tercio de las revistas incluidas en la categoría ISLS ${ }^{1}$ del WOS son totalmente ajenas a los investigadores y profesionales de la ByD. Prácticamente ningún profesional las emplea como vehículo de comunicación para trasladar sus resultados de investigación o como medio de información para mantenerse al día de los avances de su campo. Los datos no admiten duda:

-El porcentaje de autores con filiación profesional en $\mathrm{ByD}$ que publican en el citado tercio de revistas es insignificante.

-Ninguna de las referidas revistas aparece entre las citadas por los profesionales de la información o por las publicaciones propias del área. Basta con hojear en el JCR la lista de publicaciones citadas por las revistas de ByD para comprobar que ni siquiera figuran. Las mismas tablas de revistas citadas incluidas en este trabajo y en los artículos de Scimago lo demuestran palpablemente.

Como última prueba de lo ajenas que son a la $\mathrm{ByD}$, a pesar de que estén clasificadas en esta categoría en el WOS, basta con analizar cuáles son las revistas más relacionadas con ellas mismas. El propio WOS ha introducido un indicador (Related journals en los Journal citation reports) que, a partir del recuento de las revistas citantes y citadas por una publicación, permite averiguar las más cercanas. Pues bien, mírense los listados de revistas relacionadas con las susodichas 16 revistas y 


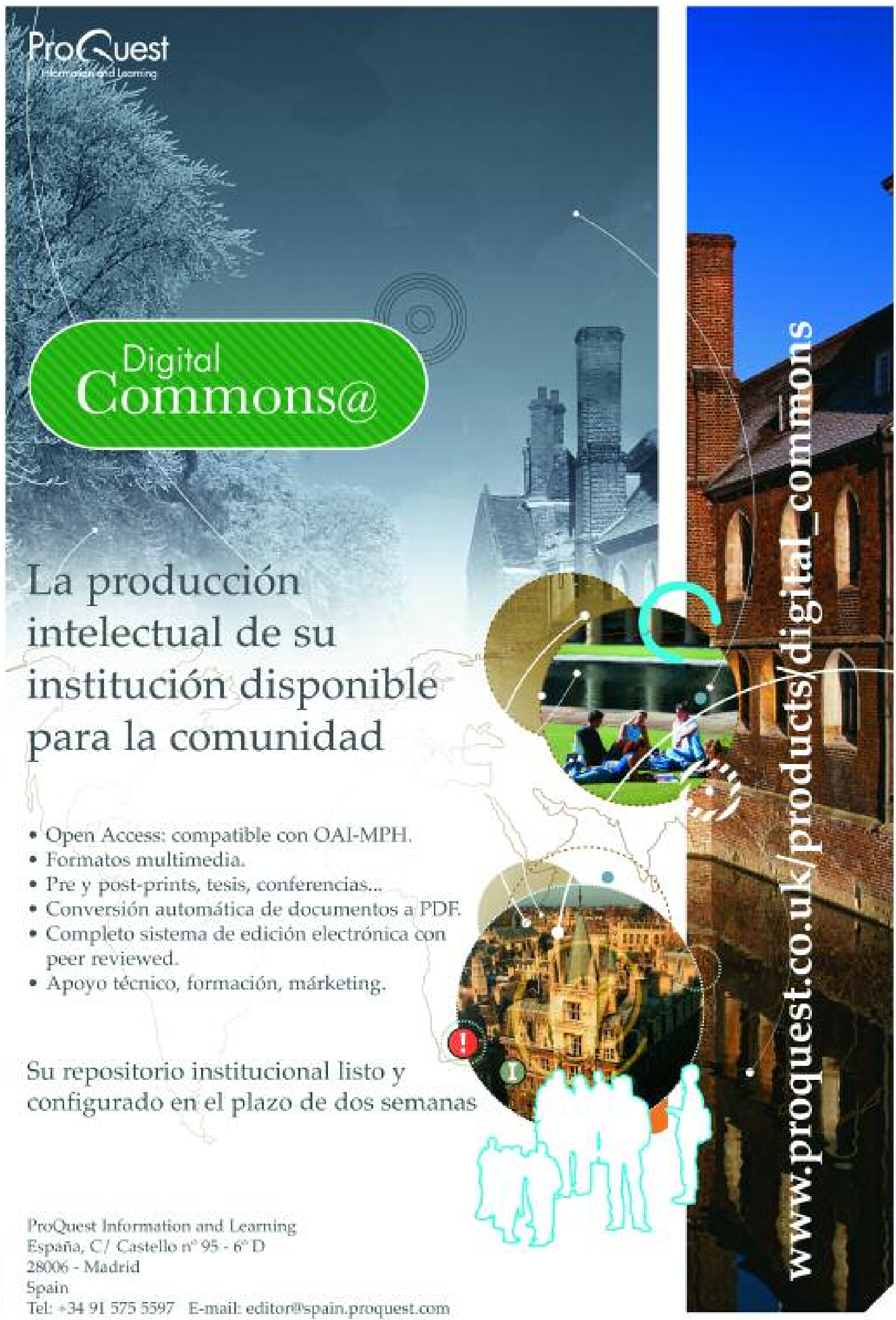


se comprobará cómo prácticamente ninguna de las que aparecen están indizadas en la propia categoría; y, por supuesto, no aparece ninguna de las revistas nucleares de la ByD (Journal of documentation, Jasist, Scientometrics, Information processing \& management, Journal of information science, Library trends, etc.). Igualmente, si se repasan los listados de revistas relacionadas con las publicaciones nucleares de la ByD se observará cómo tampoco se encuentran.

\section{Realmente lo que cuenta es el tema del artículo}

En último extremo, lo que en el fondo pudiera anidar en un planteamiento metodológico que sacraliza la categoría temática de una revista para analizar la producción científica de toda una disciplina, es la evidente confusión en lo referente a cuál debe ser la unidad de análisis de un trabajo bibliométrico de estas características. Pues bien, la unidad de análisis de estos estudios no puede ser otra que la constituida por los artículos y no las revistas, y menos aún -cual es el caso al que aquí nos referimos- las categorías temáticas de una base de datos. Incluso la posibilidad de abordar la clasificación de los artículos a través de las referencias invocada por los autores a partir de un trabajo de Glanzel et al. (1999) es expresamente cuestionada para la ciencias sociales por el propio Glanzel (2003) en un análisis posterior en el que mantiene que "it is suggested that in the social sciences the method should be used in combination with other means of subject assignment". Además en este trabajo las categorías de las que se habla no son las del ISI sino macro categorías del tipo: agriculture, biology, bio-sciences y social sciences.

Todas estas reflexiones y principios metodológicos, que no deben ser ignorados cuando se aborda el estudio de la producción científica de cualquier disciplina, deben estar aún más presentes en el caso de la ByD, debido a sus especiales señas de identidad epistemológica. Su carácter instrumental y multidisciplinar la convierten en un área muy porosa y con unas fronteras poco marcadas, siendo muy frecuente que sus conocimientos se diseminen por los más variados campos del conocimiento y que sus herramientas sean empleadas por personas de las más variadas adscripciones.

De todo lo anterior se deduce que es muy probable que artículos de ByD se publiquen en alguna de las 8.800 revistas que actualmente componen el WOS y que no pertenecen a ISLS, o que en las mismas publicaciones adscritas a esta categoría se publiquen trabajos con temáticas distintas a la propia ByD.

En conclusión, la utilización de una estrategia de búsqueda que pone como eje metodológico la categoría temática del WOS conduce a expulsar de la ByD a todos aquellos que trabajan en esta disciplina pero que publican en revistas ajenas a la misma (cosa muy frecuente sobre todo en el ámbito biomédico), o a aquellos que perteneciendo a las más variopintas filiaciones investigan sobre temas de ByD y publican fuera de las revistas nucleares de este área. Este método oculta, silencia y hace invisible parte de la producción en ByD, especialmente la ejecutada en campos de conocimiento distintos a ella.

\section{La ByD es transversal y multisectorial}

Y la prueba de que esto es así, no es sino la resultante de comparar, de un lado, los 258 trabajos localizados por Scimago para el período 1995-2004 con los 291 que ya identificara Jiménez (2002) para 1992-2001 etapa donde, por la propia dinámica de la ciencia española, la producción internacional era menor, y de otro, con los 406 (prácticamente el doble) que hemos podido identificar para el mismo intervalo temporal estudiado por Scimago. Al cubrir sólo los artículos indizados o que más citan a la categoría ISLS, la estrategia del Grupo Scimago identifica, sin duda, la producción científica nuclear de la ByD pero margina todo lo publicado fuera de estos muros, y que viene a significar prácticamente el $50 \%$ de la producción científica total de la ByD; dato que confirma el carácter multidisciplinar e instrumental de nuestra área. Una prueba más que avala este carácter instrumental es que más de un tercio de los autores responsables de dicha producción trabajan en instituciones no ligadas directamente a la ByD.

\section{«Casi un tercio de las revistas incluidas en la categoría ISLS del WOS son totalmente ajenas a los investigadores y profesionales de la ByD»}

A pesar de todo lo dicho es razonablemente posible afrontar el estudio de una o varias instituciones, especialmente si éstas son multidisciplinares, mediante la clasificación de sus aportaciones a través de las categorías WOS. Digamos que se asume que la preclasificación enviará los trabajos a las categorías más idóneas en un elevado porcentaje de los casos o a otras cercanas en los demás y que los sesgos no afectarán ni al volumen total localizado ni a los rasgos generales de estructura de especialidades; además, en el caso de estudios comparativos, los sesgos serán los mismos para todas las instituciones. Pero si se trata de un estudio enfocado a una sola especialidad, las limitaciones descritas son inasumi- 


\begin{tabular}{|c|c|c|c|c|c|c|}
\hline Autores & $\begin{array}{l}\text { Trabajos } \\
\text { publicado } \\
\text { s en } \\
\text { revistas } \\
\text { WOS } \\
\end{array}$ & $\begin{array}{c}\% \\
\text { variación } \\
\text { respecto } \\
\text { datos } \\
\text { Scimago } \\
\end{array}$ & \begin{tabular}{|c|} 
Citas a \\
trabajos \\
publicados en \\
revistas \\
WOS \\
\end{tabular} & $\begin{array}{c}\% \\
\text { variación } \\
\text { respecto } \\
\text { datos } \\
\text { Scimago } \\
\end{array}$ & $\begin{array}{l}\text { Citas } \\
\text { totales }\end{array}$ & $\begin{array}{c}\% \\
\text { variación } \\
\text { respecto } \\
\text { datos } \\
\text { Scimago } \\
\end{array}$ \\
\hline Gómez Caridad, I. & 19 & 18,8 & 101 & 16,1 & 144 & 65,5 \\
\hline Bordons Gangas, M. & 21 & 31,3 & 118 & 37,2 & 140 & 62,8 \\
\hline Zulueta, M. A. & 11 & 37,5 & 93 & 43,1 & 95 & 46,2 \\
\hline Camí Morell, J. & 7 & 75,0 & 73 & 108,6 & 83 & 137,1 \\
\hline Fernández Muñoz, M. T. & 11 & 10,0 & 75 & 1,4 & 76 & 2,7 \\
\hline Campanario, J. M. & 10 & 66,7 & 54 & 92,9 & 68 & 142,9 \\
\hline Moya Anegón, F. de & 17 & 0,0 & 36 & 9,1 & 46 & 39,4 \\
\hline Guerrero Bote, V. & 12 & 0,0 & 33 & 6,5 & 38 & 22,6 \\
\hline Maojo, V. & 13 & 225,0 & 37 & 428,6 & 37 & 428,6 \\
\hline Delgado López-Cózar, E. & 9 & 80,0 & 21 & 90,9 & 34 & 209,1 \\
\hline Méndez Miaja, A. & 4 & 0,0 & 33 & 10,0 & 33 & 10,0 \\
\hline Pulgarín Guerrero, A. & 9 & 0,0 & 30 & 7,1 & 32 & 14,3 \\
\hline Álvarez, P. & 7 & 75,0 & 27 & $-3,6$ & 28 & 0,0 \\
\hline Jiménez Contreras, E. & 10 & 42,9 & 25 & 66,7 & 27 & 80,0 \\
\hline Rey Rocha, J. & 13 & 18,2 & 21 & 10,5 & 24 & 26,3 \\
\hline Aedo, I. & 11 & 175,0 & 19 & 171,4 & 24 & 242,9 \\
\hline Sanz Casado, E. & 5 & 0,0 & 16 & 0,0 & 22 & 37,5 \\
\hline Martín Sempere, M. J. & 10 & 11,1 & 17 & 0,0 & 20 & 17,6 \\
\hline Herrero Solana, v. & 9 & 12,5 & 14 & 0,0 & 19 & 35,7 \\
\hline Llorens, J. & 7 & 16,7 & 6 & 0,0 & 16 & 166,7 \\
\hline López Pujalte, C. & 5 & 25,0 & 10 & 11,1 & 13 & 44,4 \\
\hline Plaza Gómez, L. & 8 & 33,3 & 12 & 50,0 & 13 & 62,5 \\
\hline Pinto Molina, M. & 6 & 20,0 & 9 & 50,0 & 11 & 83,3 \\
\hline Fernández Cano, A. & 5 & 25,0 & 2 & 0,0 & 10 & 400,0 \\
\hline Díaz, I. & 8 & 100,0 & 7 & 0,0 & 9 & 28,6 \\
\hline Fernández Molina, J. C. & 6 & 0,0 & 6 & 0,0 & 9 & 50,0 \\
\hline Guerra, R. A. & 4 & 0,0 & 5 & 25,0 & 6 & 50,0 \\
\hline Vives, J. M. G. & 4 & 0,0 & 5 & 25,0 & 5 & 25,0 \\
\hline Faba Pérez, C. & 3 & $-25,0$ & 3 & $-40,0$ & 3 & $-40,0$ \\
\hline López Huertas, M. J. & 5 & 25,0 & 1 & 0,0 & 2 & 100,0 \\
\hline
\end{tabular}

Tabla 3. Autores españoles más productivos y visibles en ByD en la base de datos Web of science (1995-2004)

bles y afectan gravemente a los resultados.

Sólo una estrategia de búsqueda que atienda al tema de los artículos y que cubra todos los ítems publicados en la base de datos WOS permitirá encontrar toda la producción de ByD indizada o, al menos, podrá aspirar a conseguirlo. Y eso fue lo que hizo Jiménez en 2002 y lo que se ha hecho ahora con los resulta- dos que particularizamos a continuación. Para ilustrar las diferencias con los del grupo Scimago vamos a centrarnos exclusivamente en los puntos más vidriosos de los mismos: en primer lugar en la lista de autores (tabla 3 ).

Pruebas de una evidencia

$\mathrm{Si}$ realizamos una búsqueda personalizada por autores, o sea, una consulta "tradicional" y se compara (tabla 3) con los resultados obtenidos en el mencionado trabajo de Scimago las discrepancias alcanzan proporciones preocupantes. Limitándonos a los autores identificados como más productivos, hemos podido recuperar un $28 \%$ más de trabajos y un $30 \%$ más de citas, que se elevan por encima del $50 \%$ si se consideran las dirigi- 
das a trabajos publicados en fuentes no WOS, pero citados desde esta base de datos (que es lo que se declara en el título del mencionado estudio). Se ha respetado el listado de autores incluido por Scimago y se ha introducido el porcentaje de variación en trabajos y citas de cada uno de ellos respecto al mencionado estudio, sombreando aquellos donde la variación es inexistente o mínima. Ni que decir tiene que considerar las diferencias en los apellidos o los errores en la citación, tan conocidos en esta base de datos, parece una precaución elemental $\mathrm{y}$, por tanto, han sido aspectos técnicos tenidos en cuenta.

Si comparamos los resultados del estudio de Scimago con los obtenidos en nuestra búsqueda resulta interesante subrayar ciertos cambios:

En primer lugar, de entre los autores que ya aparecían en la lista original cabe señalar que tan sólo 8 no experimentan modificación alguna frente a 17 (casi el 60\%) que ven alterada significativamente su producción o sus citas, o ambas al mismo tiempo.

En segundo lugar, destacar que los mayores cambios se concentran precisamente en los autores procedentes de áreas distintas a la ByD (Maojo, Aedo, Camí, Campanario, Díaz, Fernández Cano) o en aquellos que han publicado con frecuencia en revistas ajenas a la ByD, especialmente del área biomédica (Gómez Caridad, Zulueta, Bordons, Delgado López-Cózar y Jiménez Contreras). A esta relación habría que añadir la incorporación de investigadores ausentes en la lista de Scimago como los de Herrera Viedma, E. (10 trabajos); De Campos, L. M.; Fernández Luna, J. M.; Huete, J. F. (9); Buela Casal, G. (8); Martín Sánchez, F. (7); Ruiz Pérez, R. (5); García Figuerola, C.; Barrigón, S.; Vila, M. A. y Cordón, O. (4), etc., por citar tan sólo a algunos de los que aparecen con producciones más elevadas, lo que subraya uno de los aspectos más relevantes de ByD: la presencia significativa y continua de científicos no vinculados a la misma que es muy superior al marginal $10 \%$ descrito por el trabajo al que nos referimos.

En tercer lugar, y como consecuencia de estos recuentos, hay también cambios generalizados en las posiciones, algunos tan apreciables como la inversión de los autores primero y segundo entre sí (en cuanto a producción) y el tercero y cuarto. Ello es de escasa significación estadística en sí mismo pero de mucha importancia personal para los afectados. En líneas generales todos los autores, excepto dos, experimentan cambios de posición, algunos llamativos: J. M. Campanario del 10 al 6; V. Maojo del 22 al 8. Hay muy pocos casos en que los autores vean inalteradas sus cifras de producción y sólo uno que retroceda (C. Faba Pérez, que de hecho no debería aparecer en la tabla).

\section{«Podemos afirmar que la repercusión y visibilidad \\ internacional de la producción científica en ByD es bastante deficiente»}

Dado que estamos tratando con personas con una trayectoria investigadora, nos parece necesario mantener el más escrupuloso respeto a su labor y proceder con la mayor cautela a fin de que, en la medida de lo posible, no quede por el camino ni una sola de sus aportaciones y ni una sola de sus citas; en este orden de cosas creemos que el nuevo cuadro que hemos descrito se ajusta más a la realidad investigadora de nuestra especialidad y aun así no hace justicia a los autores que deberían aparecer en esta tabla y que no lo hacen por no haber alcanzado en el trabajo que da origen a esta réplica el umbral mínimo de 4 trabajos. Dejamos para una próxima ampliación el pago de esta deuda.

\section{Endocitas: influimos poco fuera de España}

Por otra parte, puesto que nuestro objetivo es detectar la visibilidad y la repercusión internacional de la producción española en ByD, es de suma importancia identificar qué porcentaje del total de citas recibidas por un autor son autocitas. Cuando se trata de establecer un escalafón deben excluirse de los recuentos, que es, por otra parte, lo habitual en los estudios bibliométricos de esta guisa. Y así lo hicieron Moya y Jiménez (1999), en un estudio anterior de similares características publicado en esta misma revista. En la tabla 4 los autores españoles más productivos, de acuerdo con los datos de Scimago, aparecen ordenados por las citas recibidas menos sus autocitas a fin de presentarlos en función de la atención que sus trabajos han merecido entre los restantes investigadores de la especialidad. Al mismo tiempo, y a fin de tener un retrato más fiel y ajustado del impacto de los autores, se incluye el porcentaje de autocitación de cada autor. Se han sombreado aquellos cuya tasa de autocitación es superior al $70 \%$.

A efectos metodológicos y dada la presencia habitual de grupos de autores en la firma de los trabajos, es preciso señalar que se ha considerado como autocita toda aquella en que un autor, cualquiera que sea su posición en el orden de firma, cita un trabajo en el que también ha participado, independientemente -también en este caso- de su posición como firmante.

A la vista de los datos de citación alojados en la tabla 4 podemos afirmar que la repercusión y visibilidad internacional de la producción científica en ByD es bastante deficiente. No sólo el número de citas 


\section{¿Es la gestión de su colección electrónica}

\section{más complicada de lo que esperaba?}

\section{Nosotros le podemos ayudar.}

Conseguir el listado exacto de los titulos que ha suscrito y asi poder gestionar correctamente el registro o solucionar los problemas de acceso a sus revistas y libros electrónicos, puede requerir más tiempo del que usted dispone.

Desde EBSCO gestionamos sus compras individuales o consorciadas de paquetes e, incluyendo un listado exacto de las tevistas y librose suscritos, facturas detalladas para facilitar las asignaciones de presupuestos y gestión personalizada de los informes de colecciones que le ayudarản en el desarrollo de su colección.

Nuestro equipo de servicio al cliente está a su disposición para ayudarle a resolver los problemas de acceso, los cambios en las direcciones IP, etc- Las herramientas de gestión de recursos-e de EBSCO han sido creadas con el fin de minimizar las tareas administrativas al tiempo que los usuarios maximizan sus resultados.

Ponemos toda nuestra información y experiencia a su disposición.

Contacte con su representante de FBSCO. 
brutas es bajo sino que la tasa de autocitación es muy elevada. El $47 \%$ son autocitas, lo que significa que una de cada dos citas que recibe un trabajo español de ByD en el $W O S$ es realizada por el propio autor. Sólo dos autoras consiguen apenas alcanzar la cifra de las 100 citas externas; sin embargo, más de la mitad de los que aparecen en la tabla poseen 10 o menos citas, lo que vendría a representar una por año. Ahora bien, existen acusadas diferencias entre autores.

Atendiendo al número total, descontadas las autocitas, y a la tasa de autocitación, pueden identificar-

\begin{tabular}{|l|c|c|c|c|}
\hline \multicolumn{1}{|c|}{ Autores } & Citas & Autocitas & $\begin{array}{c}\text { Citas sin } \\
\text { autocitas }\end{array}$ & $\begin{array}{c}\% \text { de } \\
\text { autocitas }\end{array}$ \\
\hline Gómez Caridad, I. & 136 & 36 & 100 & 26,5 \\
\hline Bordons Gangas, M. & 137 & 41 & 96 & 29,9 \\
\hline Zulueta, M. A. & 89 & 22 & 67 & 24,7 \\
\hline Cami Morell, J. & 83 & 16 & 67 & 19,3 \\
\hline Fernández Muñoz, M. T. & 72 & 10 & 62 & 13,9 \\
\hline Campanario, J. M. & 68 & 14 & 54 & 20,6 \\
\hline Méndez Miaja, A. & 33 & 2 & 31 & 6,1 \\
\hline Delgado López-Cózar, E. & 34 & 15 & 19 & 44,1 \\
\hline Sanz Casado, E. & 22 & 4 & 18 & 18,2 \\
\hline Maojo, V. & 30 & 14 & 16 & 46,7 \\
\hline Jiménez Contreras, E. & 24 & 8 & 16 & 33,3 \\
\hline Rey Rocha, J. & 30 & 15 & 15 & 50,0 \\
\hline Pulgarín Guerrero, A. & 30 & 19 & 11 & 63,3 \\
\hline Álvarez, P. & 28 & 17 & 11 & 60,7 \\
\hline Aedo, I. & 24 & 14 & 10 & 58,3 \\
\hline Pinto Molina, M. & 17 & 7 & 10 & 41,2 \\
\hline Plaza Gómez, L. & 14 & 4 & 10 & 28,6 \\
\hline Guerrero Bote, V. & 39 & 31 & 8 & 79,5 \\
\hline Fernández Cano, A. & 10 & 10 & 7 & 30,0 \\
\hline Herrero Solana, V. & 28 & 21 & 7 & 75,0 \\
\hline Martín Sempere, M. J. & 20 & 14 & 6 & 70,0 \\
\hline Moya Anegón, F. de & 51 & 46 & 5 & 90,2 \\
\hline Guerra, R. A. & 6 & 1 & 5 & 16,7 \\
\hline Llorens, J. & 24 & 20 & 4 & 83,3 \\
\hline López Pujalte, C. & 14 & 10 & 4 & 71,4 \\
\hline Díaz, I. & 7 & 3 & 4 & 42,9 \\
\hline Vives, J. M. G. & 5 & 6 & 4 & 20,0 \\
\hline Fernández Molina, J. C. & 3 & 0 & 100,0 \\
\hline López Huertas, M. J. & 1 & 1 & 75,0 \\
\hline Faba Pérez, C. & 3 & 16,0 \\
\hline
\end{tabular}

Tabla 4. Citas a autores españoles de ByD más productivos y visibles en la base de datos Web of science (1995-2004) tradicional carácter abierto de la ByD. El segundo, con un número de citas oscilante entre 15 y 30 y con tasas de autocitación todavía inferiores a la media (30 a 45\%), está formado mayoritariamente por profesores procedentes de la universidad (Elías Sanz, Maojo, Delgado López-Cózar, Jiménez Contreras) y por otros miembros del Cindoc (Aida Méndez, jubilada hace varios años, y Jesús Rey). El tercer grupo, estaría conformado por aquellos autores con un número de citas inferior a 11 y con tasas de autocitación, salvo excepciones, superiores al 60\%; la mayoría están ligados a la universidad.

Un dato que resulta llamativo aunque no sorprendente es que, en líneas generales, y con alguna que otra excepción, aquellos autores con más citas son los que presentan una tasa de autocitación menor; en cambio, los que poseen un número menor de citas son los que mayores tasas de autocitación poseen, lo cual quiere decir que sus citas y su repercusión científica dependen de ellos mismos.

\section{«Podríamos \\ hablar de la paradoja española, aquella que consiste en publicar fuera para ser citado dentro»}

Sin querer ser excesivamente pesimistas debemos reconocer que el reflejo científico internacional de la ByD española, medida en términos de citas en la base de datos WOS, es más que reducido. Si a las autocitas sumáramos las citas procedentes de autores domésticos, entendiendo por tales a las generadas por autores españoles, constataríamos que nuestra auténtica repercusión internacional de la ByD es ca- 
si nula, algo que no es diferente a lo que ocurre en otros sectores científicos aunque sí se da en este con mayor intensidad. Así podríamos hablar de la paradoja española, aquella que consiste en publicar fuera para ser citado dentro. En definitiva, se plantea este inquietante interrogante: $i$ es posible que los trabajos de autores españoles sólo hayan sido leídos por otros que no sean ellos mismos o sus colegas más próximos? Puede ser, pues aunque sabemos que no todo lo que se lee se cita y que no todo lo que se cita se ha leído, la realidad es que los trabajos firmados por autores españoles no consiguen, por la razón que sea, ser citados fuera de su círculo más íntimo. No obstante este es un tema que por su envergadura merece un tratamiento monográfico, que dejamos para otro momento.

\section{Muchas más revistas a considerar}

En cuanto a los listados de revistas $W O S$ en las que han publicado los autores españoles (tabla 5) y los de revistas citadas por ellos (tabla 6) afloran también importantes cambios respecto a los resultados ofrecidos por el trabajo de Scimago (se han sombreado las revistas en las que se constatan mayores diferencias). Así, mientras que aquí se han localizado 171 revistas en las que se han publicado trabajos de $\mathrm{ByD}$, en el análisis de Scimago se localizaban sólo 68 (tabla 5). Pero las diferencias más acusadas se producen en las posiciones ocupadas. El caso más espectacular es el de Medicina clínica (una fuente externa al área) que aparecía con cuatro artículos y que se encarama hasta la segunda posición de la lista de revistas con 31; Arbor, que figura en la sección del $A \& H C I$ del $W O S$ con 11, equipara en producción a algunas de las revistas nucleares de la ByD.

El aspecto más visible es la presencia de revistas biomédicas en

\begin{tabular}{|c|c|c|}
\hline Revistas & $\begin{array}{l}\text { Trabajos } \\
\text { publicados } \\
\text { sobre ByD }\end{array}$ & $\begin{array}{c}\text { Trabajos } \\
\text { según estudio } \\
\text { Scimago } \\
\end{array}$ \\
\hline Scientometrics & 48 & 48 \\
\hline Medicina clínica & 31 & 4 \\
\hline $\begin{array}{l}\text { Journal of the American Society for } \\
\text { Information Science and Technology } \\
\text { (anteriormente Journal of the American } \\
\text { Society for Information Science) }\end{array}$ & 23 & 23 \\
\hline Information processing \& management & 13 & 13 \\
\hline Journal of information science & 11 & 11 \\
\hline Arbor-ciencia pensamiento y cultura & 11 & 2 \\
\hline Libri & 8 & 8 \\
\hline Journal of documentation & 8 & 8 \\
\hline Research evaluation & 7 & 7 \\
\hline Electronic library & 7 & 7 \\
\hline Revista de neurología & 7 & 2 \\
\hline Revista española de cardiología & 7 & 2 \\
\hline $\begin{array}{l}\text { International journal of approximate } \\
\text { reasoning }\end{array}$ & 7 & 0 \\
\hline $\begin{array}{l}\text { Information research-an international } \\
\text { electronic journal }\end{array}$ & 6 & 6 \\
\hline Information technology and libraries & 5 & 5 \\
\hline Knowledge organization & 5 & 5 \\
\hline Online information review & 5 & 5 \\
\hline Interciencia & 5 & 3 \\
\hline Psicothema & 5 & 0 \\
\hline Archivos de bronconeumología & 4 & 0 \\
\hline Nature & 4 & 0 \\
\hline $\begin{array}{l}\text { Evaluation of cross-language } \\
\text { information retrieval systems }\end{array}$ & 4 & 0 \\
\hline $\begin{array}{l}\text { International journal of uncertainty } \\
\text { fuzziness and knowledge-based } \\
\text { systems }\end{array}$ & 4 & 0 \\
\hline Advances in information retrieval & 4 & 0 \\
\hline Psicología conductual & 4 & 0 \\
\hline Online \& cdrom review & 3 & 3 \\
\hline Neurología & 3 & 0 \\
\hline $\begin{array}{l}\text { Revista española de enfermedades } \\
\text { digestivas }\end{array}$ & 3 & 0 \\
\hline $\begin{array}{l}\text { Advances in cross-language information } \\
\text { retrieval }\end{array}$ & 3 & 0 \\
\hline Advances in web intelligence & 3 & 0 \\
\hline Biosystems & 3 & 0 \\
\hline Pattern recognition & 3 & 0 \\
\hline 31 títulos con 2 trabajos & 62 & \\
\hline 106 títulos con 1 trabajo & 106 & 31 \\
\hline
\end{tabular}

Tabla 5. Revistas indizadas en la base de datos Web of science en que publican autores españoles trabajos de ByD (1995-2004) 
posiciones destacadas: Revista de neurología, Revista española de cardiología, etc., lo que viene a demostrar el importante papel que la documentación médica ha jugado (y sigue haciéndolo) dentro de la ByD española (Delgado López-Cózar, 2003). También cabe resaltar el importante número de revistas ligadas a las ciencias de la computación, dado que la tecnología de los procesos de almacenamiento y recuperación de información tiene una base informática que hace necesaria la presencia de medios de comunicación de este área. También es reseñable la aparición de revistas ligadas a la psicología, que es otra constante en la documentación española. Por último cabe subrayar como los dos sistemas de búsqueda coinciden a la hora de identificar cuáles son las revistas de la categoría ISLS del WOS que pueden considerarse nucleares en la ByD (Scientometrics, Jasist, Journal of documentation, Information processing \& management, Journal of information Science, Libri, etc.) y cuáles no (entre ellas las 16 listadas con anterioridad, que no consiguen atraer trabajos de autores españoles que laboran en las instituciones documentales).

Por lo que respecta a las revistas citadas, los cambios son evidentes y generalizados. Todas las ya identificadas por Scimago cambian los valores, y en todas se produce un aumento a la recuperación de citas. En la mayoría de los casos los valores se multiplican por dos. El cambio más trascendente es la posición destacada que ocupa Medicina clínica, que se convierte en la más citada por los autores españoles de ByD a nivel internacional. Este resultado está en sintonía absoluta con lo hallado por Mercedes de la Moneda (2003) en su análisis bibliométrico de la producción bibliográfica española en ByD (1984-1999). En dicho período era la revista más citada de la ByD es-

\begin{tabular}{|c|c|c|}
\hline Revistas & $\begin{array}{c}\text { Trabajos } \\
\text { publicados } \\
\text { sobre ByD }\end{array}$ & $\begin{array}{c}\text { Trabajos } \\
\text { según estudio } \\
\text { Scimago }\end{array}$ \\
\hline Medicina clínica-Barcelona & 406 & 267 \\
\hline Scientometrics & 405 & 170 \\
\hline $\begin{array}{l}\text { Journal of the American Society of } \\
\text { Information Science and Technology }\end{array}$ & 380 & 177 \\
\hline Journal of documentation & 99 & 51 \\
\hline Information processing \& management & 94 & 38 \\
\hline $\begin{array}{l}\text { Revista española de documentación } \\
\text { científica }\end{array}$ & 94 & 68 \\
\hline Journal of information science & 85 & 61 \\
\hline Nature & 80 & 26 \\
\hline British medical journal & 78 & \\
\hline Science & 77 & 35 \\
\hline Communication ACM & 76 & 18 \\
\hline Information retrieval & 66 & \\
\hline Information processing & 53 & 26 \\
\hline Lancet & 45 & \\
\hline Journal of American Medical Association & 41 & \\
\hline Research evaluation & 39 & 29 \\
\hline Archivos de bronconeumología & 37 & \\
\hline Research policy & 36 & 15 \\
\hline Library trends & 35 & 24 \\
\hline Annual review information science & 32 & \\
\hline Lectures notes in artificial intelligence & 31 & \\
\hline Current contents & 27 & 35 \\
\hline IEEE transactions pattern analysis & 25 & \\
\hline Patterns recognition & 25 & \\
\hline International journal intelligent systems & 24 & \\
\hline Revista española de cardiología & 24 & \\
\hline Atención primaria & 23 & \\
\hline Revista de neurología & 23 & 2 \\
\hline Annals internal medicine & 22 & \\
\hline Automatic test processing & 22 & \\
\hline Bulletin Medical Library Association & 22 & 0 \\
\hline New England journal of medicine & 22 & \\
\hline American psychologist & 21 & \\
\hline ACM Transact on information systems & 20 & \\
\hline Artificial intelligence & 20 & \\
\hline Lecture notes in computer science & 20 & \\
\hline
\end{tabular}

Tabla 6. Revistas más citadas por los trabajos publicados por autores españoles en revistas indizadas en la base de datos Web of science (1995-2004)

pañola. En 9 de los 13 años en que se calcularon índices de repercusión figuraba como la de mayor impacto. Junto a ella aparecen algunas de las más prestigiosas revistas biomédicas del mundo (British medical journal, Journal of the American Medical Association, The lan- cet) y las mismas revistas biomédicas españolas que ya eran importantes como canales de publicación. Ocupan también puestos relevantes Nature y Science que son las publicaciones multidisciplinares por excelencia. El cuadro se completa con la aparición de otras de carácter in- 


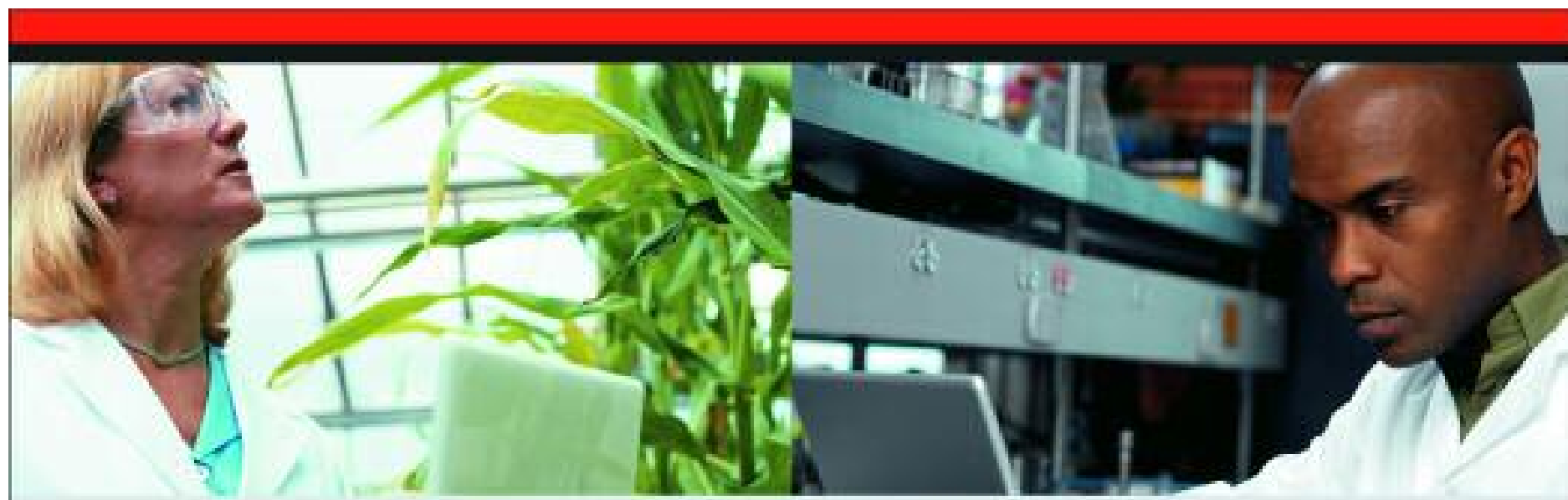

nature.com es ciencias naturales

nature.com es ciencias físicas

\title{
Una licencia de sitio es su acceso a nature.com
}

\author{
Efectuando su pedido en 2006 su institudión accederá a los archivos completos de la revista. \\ Póngase en contacto con su representante pera más información sobre nuestra nueva politica de \\ cancelacicn y sobre las modificaciones del contrato de licencia en 2007
}

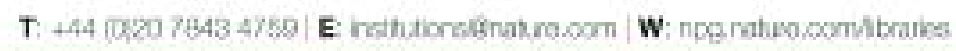

formático que vuelven a revelar los especiales lazos entre la ByD y las ciencias de la computación. Y, por último, de nuevo recalcar como las revistas ByD que se encuentran encabezando el listado constituyen las fuentes intelectuales de referencia de la ByD española (Scientometrics, Jasist, Journal of documentation, Information processing \& management, Journal of information science). A este núcleo se incorpora la Revista española de documentación científica.

\section{Notas}

1. Government information quarterly, Information \& management, International journal of geographical information science, Information society, Information systems journal, Information systems research, International journal of information management, Journal of The American Medical Informatics Association, Journal of government information, Journal of health communication, Journal of information technology, Journal of management information systems, Mis quarterly, Social science computer re- view, Social science information, Telecommunications policy.

2. Aunque actualmente la profesora Zulueta trabaja como docente de la Universidad de $\mathrm{Al}$ calá de Henares, debe incluirse en el Cindoc, pues es en esta institución donde ha desarrollado su trayectoria como investigadora y donde ha producido la mayoría de los trabajos que son objeto de este estudio.

\section{Bibliografía}

Delgado López-Cózar, E. La investigación en biblioteconomía y documentación. Gijón: Ediciones Trea, 2003. ISBN 84-9704-041-4.

Glanzel, W; Schubert, A. "A new classification scheme of science fields and subfields designed for scientometric evaluation purposes". En: Scientometrics, 2003, v. 56, n. 3, pp. 357-367.

Glanzel, W.; Schubert, A.; Czerwon, H. J. "An item-by-item subject classification of papers published in multidisciplinary and general journals using reference analysis". En: Scientometrics, 1999, v. 44, n. 3, pp. 427-439.

Jiménez Contreras, E. "La aportación española a la producción científica internacional en biblioteconomía y documentación: balance de 10 años (1992-2001)". En: BiD, Textos universitaris en biblioteconomia i documentació, 2002, n. 9. Consultado el 26-05-06.

http://www.ub.es/bid/bid09.htm
Moneda Corrochano, M. Análisis bibliométrico de la producción bibliográfica española en biblioteconomía y documentación, 1984-1999. Granada: Universidad de Granada: Departamento de Biblioteconomía y Documentación, 2003.

Moya Anegón, F.; Jiménez Contreras, E. «Evolución de la producción científica española de los últimos 25 años». En: El profesional de la información, 1999, v. 8, n. 5, pp. 26-29.

Scimago. «Producción española con visibilidad internacional (ISI-WOS) en biblioteconomía y documentación (I)». En: El profesional de la información, 2005, v. 14, n. 6, pp. 459-461.

Scimago. «Producción española con visibilidad internacional (ISI-WOS) en biblioteconomía y documentación (II)». En: El profesional de la información, 2006, v. 15, n. 1, pp. 34-36.

\section{Evaristo Jiménez Contreras, Emilio} Delgado López-Cózar y Rafael Ruiz Pérez, EC : Evaluación de la Ciencia y de la Comunicación Científica, Departamento de Biblioteconomía y Documentación, Universidad de Granada, Campus de Cartuja, 18071 Granada. evaristo@ugr.es edelgado@ugr.es rruiz@ugr.es 